\title{
Wire Haired Standard Dachshund
}

National Cancer Institute

\section{Source}

National Cancer Institute. Wire Haired Standard Dachshund. NCI Thesaurus. Code C53905.

The Wire Haired Standard Dachshund is a wire-haired variety of dachshund with a standard height up to 9 inches. 